\title{
XXXIII.
}

\section{Ueber die Veränderungen des Gehirns und Rückenmarks bei Lyssa.}

\author{
Von \\ Dr. 0. Weller, \\ Secundararzt an der Irrenanstalt Burghölzli bei Zürich.
}

(Hierzu Taf. VII.)

\begin{abstract}
Die pathologische Anatomie kannte bis in die neueste Zeit keine Veränderungen der Nervencentren, welche die foudroyanten Krankheitserscheinungen bei der Wuthkrankheit zu erklären im Stande gewesen wären. Noch im Jahre 1876 geben Röll*) und ebenso Bollinger**), allerdings mit Nichtberücksichtigung einiger indessen erschienener Arbeiten, als einzigen positiven Sectionsbefund bei Lyssa, Hyperämie des Gehirns und seiner Häute an.

In den Jahren 1874 und 75 veröffentlichte Benedikt***) das Resultat einer Reihe pathologisch-anatomischer Untersuchungen, welche er an den Nervencentren eines an Lyssa gestorbenen Menschen und einer Anzahl dieser Krankheit erlegener Hunde vorgenommen hat. Er beschreibt, kurz resümirt, folgende pathologische Veränderungen:

1. Hyperämie und Erweiterung der Gefässe, Blutungen, Auftreten von rothen und weissen Blutkörperchen unter der Adventitia der Gefässe.

*) Lehrbuch der Pathologie und Therapie der Hausthiere von Dr. M. F. Röll. Wien 1876. (IV. Auflage.)

**) Zoonosen von Prof. 0. Bollinger im Handbuch der speciellen Pathologie u Therapie von Dr. H. v. Ziemssen III. Bd. II. Aufl. Leipzig 1876. ***) Wiener med. Presse 1874 No. 27 und Virch ow's Archiv. Bd. 64. p. 557 (Zur pathologischen Anatomie der Lyssa von Prof. Dr. Moritz Benedikt in Wien.)
\end{abstract}


2. Lymphostase im Gewebe des Gehirns, miliare, beim Menschen traubenförmig angeordnete Abscessherde und byaloide Herde in der Hirnsubstanz, Granulärdesintegration der Grundsubstanz des Gehirns und Pigmentschollen und Pigmenthüllen, welche die Gefässe wie ein Panzer umgeben.

Im Ganzen ähnliche mikroskopische Befunde beschreibt Kolesnikoff*), nämlich 1. Ausdehnung und Hyperämie der mit rothen Blutkörperchen vollgestopften Gefässe, Anhäufungen von rothen und weissen Blutkörperchen in den perivasculären Räumen, 2. Hyaloide, bisweilen sich im Lumen der Gefässe ausbreitende und Letzteres thrombosirende Massen in den Gefässwandungen und 3. Ansammlung vou runden, indifferenten Elementen um und in die Nervenzellen, wodurch das Protoplasma der Letzteren theilweise zerstört wird.

Kolesnik off's Untersuchungen erstreckten sich auf zehn Hundegehirne.

Mit den Mittheilungen Kolesnikoff's stimmen theilweise die Angaben eines andern Bearbeiters, Wassilieff's**), überein. Er fand im Gehirn einer an Lyssa verstorbenen Frau: 1. Trübung einzelner Nervenzellen des verlängerten Markes und Undeutlichkeit ihrer Contouren und Kerne; 2. Anhäufung von indifferenten runden Elementen (weissen Blutkörperchen) in dem interstitiellen Gewebe des Gehirns, insbesondere in den perivasculären Räumen; 3. Hyperämie der Blutgefässe mit stellenweiser Schwellung des Endothels und hie und da auftretender feinkörniger Umwandlung ihrer Wandungen und 4. bezeichnet er als die hervorragendste Erscheinung die Anwesenheit einer mattglänzenden, stark lichtbrechenden, hyaloiden Substanz in den perivasculären Räumen, welche sich weder in starken Säuren, noch bei Kochen mit Aetzkali, noch in Terpentin und Alkohol löste.

Ferner findet sich im Centralblatt für med. Wissenschaft 1878 p. 208 und 284 notirt, dass Gowers ${ }^{* * *}$ ) in vier von ihm untersuchten Fällen von Hydrophobie perivasculäre Zellenanhäufungen an verschiedenen Stellen des Gehirns, besonders im Hypoglossuskerne und

*) Nicolaus Kolesnikoff. Pathologische Veränderungen im Nervensystem bei der Wuthkrankheit. Centralblatt für d. med. Wissensch. 1875 . p. 853.

*:*) Dr. N. Wassilieff. Ueber die Veränderungen des Gehirns und der Herzganglien bei der Lyssa. Centralblatt für d. med. Wissensch. 1876. p. 625 .

**: ) W. R. Gowers, The pathological anatomy of hydrophobia. From Anat. pathol. Transact. 1877, S.-A. 
Chaedle*) starke Hyperämie des Pons und der Medulla oblongata und kleine Haemorrhagien in diesen Theilen beschrieben haben. Die Originalarbeiten dieser englischen Autoren standen mir nicht zu Gebote.

Diesen positiven Befunden gegenüber gelangte Fore ${ }^{* *}$ ) auf Grund von Untersuchungen, die er an den Gehirnen zweier Hunde, zweier Pferde, eines Rindes und eines Menschen, welche der Wuthkrankheit erlegen waren, anstellte, zu ziemlich negativen Resultaten. Als einzig nenneswerthe Veränderung führt er mässige Lymphzellenansammlung in den perivasculären Räumen und geringe Kernvermehrung im Gewebe der bezüglichen Organe an.

Ebenso sah Fr. Schultze ${ }^{* * *}$ ) bei einem an Lyssa verstorbenen Menschen und einem derselben Krankheit erlegenen Hunde keine Veränderungen im Centralnervensystem.

Eine in den Jahren 1875-78 im Kanton Zürich herrschende Epidemie von Lyssa canina gab mir Gelegenheit eigene Untersuchungen über das Verhalten der Nervencentren bei dieser Krankheit anzustellen. Hiebei stiess ich allerdings auf sehr bedeutende pathologische Veränderungen, welche zum Theil mit den schon Eingangs beschriebenen positiven Befunden anderer Forscher übereinstimmen. Die bezüglichen Untersuchungsobjecte erhielt ich alle bis auf eines aus der Thierarzneischule Zürich durch die gütige Vermittlung der Herren Director Zangger und Prof. Eberth. Ich untersuchte im Ganzen sieben Gehirne und Rückenmarke. Davon stammen drei aus dem Jahre 1875 (Januar, Februar, März). Dieselben wurden von mir sowohl im frischen Zustande als nach Härtung untersucht; ebenso das Gehirn eines im Sommer 1876 der Lyssa erlegenen Hundes, das mir aus Aarau zugesandt wurde. Ein im Jahre 1877 aus der Thierarzneischule Zürich erhaltenes Gehirn gelangte nur zu frischer Untersuchung. Die Härtang des schon etwas macerirten Präparates missglückte. Zwei weitere aus dem Jahre 1877 stammende Hirne and Rückenmarke wurden von mir nur in gehärtetem Zustande, dagegen von Herrn Prof. Eberth frisch untersucht. Die letzten Präparate waren in Alcohol gehärtet, die übrigen in doppeltehromsaurem Kali. Die Untersuchung aller dieser Präparate, sowohl die in frischem

*) W. B. Chaedle, A lecture on the pathology of hydrophobia. Med. Times and Gaz. 1877. No. 1432.

**) Dr. A. Forel. Ueber die Hirnveränderungen bei Lyssa. Dentsche Zeitschrift f. Thiermed. and vergl. Pathologie. III. Bd. p. 260.

***) Fr. Schultze. Zur pathologischen Anatomie der Chorea minor, des Tetanus und der Lyssa. Deutsches Aroh. f. klin. Med. 20. p. 383. 
Zustande, als nach Härtung, vorgenommene, ergab so übereinstimmende Resultate, dass ich mich füglich auf eine allgemeine Zusammenstellung: derselben beschränken darf.

A.

\section{Untersuchung in trischem Zustande.}

Bei der Betrachtung frischer Präparate fällt schon mit unbewaffnetem Auge der grosse Blutreichthum der Nervencentren auf. Nicht allein ist die Pia des Gehirus und des Rückenmarks stark hyperämisch: auch die Gefässe der Hirnsubstanz und zwar sowohl der Rinde, als der Markmasse und der grossen basalen Ganglien, sind vielfach strotzend gefüllt und auf Querschnitten der Medulla oblongata und des Rückenmarks treten die hyperämischen Gefässe als zahlreiche breite, kleine Apoplexien vortäuschende Gefässpunkte und Gefässstriemen hervor und zwar im Rückenmark vorwiegend in der grauen Substanz.

Zupft man aus der Hirnsubstenz oder aus dem Rückenmark kleine Gefässe heraus und unterwirft dieselben der mikroskopischen Betrachtung, so treten folgende auffallende, pathologische Erscheinungen zu Tage.

1. Zunächst fällt sofort wieder die Hyperämie auf. Es zeigt sich aber, dass dieselbe in den einzelnen Gefässbezirken eine verschiedene ist und auch in den einzelnen Aesten derselben Ramification sehr differirt, indem die hyperämische Füllung und Erweiterung häufig nur einen Theil der Aeste, desselben Gefässbezirks betrifft. Am ausgesprochensten ist die Hyperämie in der Medulla oblongata und in dem obern Theile des Halsmarkes und nimmt im Allgemeinen von hier sowohl nach oben, als nach unten ab.

2. In den perivasculären Räumen eines Theils der Gefässe sieht man eine mehr oder weniger starke Anhäufnng von lymphoiden Elementen. Auch hier sind bedentende Differenzen in Bezug auf die Verbreitung and Intensität der Affection zu constatiren.

Diese lymphoide Auswanderung ist da am stärksten entwickelt, wo wir auch die intensivste Hyperämie treffen - in der Medulla oblongata. Hier und in den angrenzenden Partien des Halsmarks und des Pons sind beinahe alle Gefässe bis zum Uebergang in die Capillaren mehr oder weniger davon betroffen and es ist die Anhäufung von lymphoiden Zellen häufig eine enorme. Viele Gefässe erscheinen dadurch wie von einer breiten, traubenbeerartigen Hülle umkleidet, die mitunter doppelt und dreifach so breit ist, als das Gefässlumen selbst. Die perivasculären Lymphräume der betreffenden Gefässe erleiden dadurch eine colossale Ausdehnung (Fig. 1A., Fig. 2A.). 
Je weiter wir uns nach oben hin von der Medulla oblongata entfernen, um so geringer wird im Allgemeinen die beschriebene Exsudation, sowohl in Bezug auf die Hänfigkeit des Vorkommens, als in Bezug auf Intensität der Affection. Doch ist wohl zu bemerken, dass noch viele Gefässe der Vierhügel, der Thalami optici, der Corpora striata, des Linsenkerns und des Brust- und Lendenmarks die beschriebene Veränderung in ziemlich hohem Grade zeigen. Am wenigsten ist dieselbe im Allgemeinen ausgesprochen in der Markmasse und der Rinde der Grosshirnhemisphären und im Kleinhirn, ohne jedoch daselbst vollständig zu verschwinden. Auch hier finden sich immer noch einzelne Gefässe, deren perivasculärer Raum durch eine ein- bis zweifache Schicht von Lymphelementen ausgedehnt ist.

Was die betreffenden Elemente selbst anbetrifft, so haben sie im Allgemeinen ganz das Aussehen und die Grösse weisser Blutkörperchen. Es sind rundliche bis ovale Zellen mit granulirtem Inhalt. Bei Behandlung mit Essigsäure zeigen sie einen deutlichen, relativ grossen Kern. Mitunter sieht man auch zwei deutlich abgegrenzte Kerne. Mit Carmin tingiren sie sich ziemlich rasch. Sehr schön werden sie von Hämatoxylin gefärbt. Unter ihnen befinden sich nicht selten Kerne, welche in fettigem Zerfall begriffen erscheinen, gequollen, mit undeutlichen Contouren und stark krümlig, granulärem Inhalt. Was die Natur und den Ursprung dieser lymphoiden Elemente anbetrifft, so unterliegt für mich kein $\mathrm{Zweifel,} \mathrm{dass} \mathrm{es} \mathrm{ausgewanderte}$ weisse Blutkörperchen sind; nicht allein spricht dafür ihr Aussehen, und ihre Beschaffenheit, sondern auch der Umstand, dass ihr massenhaftes Vorhandensein stets mit Hyperämie der afficirten Gefässe zusammenfällt und sie nicht selten von rothen Blutkörperchen begleitet sind.

3. Es finden sich nämlich in den perivasculären Räumen neben jenen lymphoiden Zellen $a b$ und $z u$ auch rothe Blutkörperchen und zwar bald nur vereinzelt, bald in grössern Massen angehäuft. Dieselben erscheinen ziemlich frisch, mit wenig veränderten Contouren. In Zerfall begriffene rothe Blutkörperchen habe ich nicht gesehen.

4. Was bei der mikroskopischen Untersuchung der Gefässe in frischem Zustande neben der Anhäufung von lymphoiden Elementen am meisten auffällt, ist ein massenhaft um und in die Gefässwandungen abgelagerter Fettkörper (Fig. 1B.). Derselbe bildet kreisrunde, ovale, oder polygonale, glänzende, stark lichtbrechende Schollen, mit scharf markirten Contouren von blass- bis goldgelber Färbung. Ihre Grösse variirt zwischen 0,0015-0,01 $\mathrm{Mm}$. und darüber. Dieselben verändern sich in Säuren nicht und in verdünnten Alkalien lässt sich ebenfalls 
keine merkliche Veränderung an denselben constatiren. Mit concentrirten Alkalien 12-24 Stunden stehen gelassen oder nur kurze Zeit gekocht, verwandeln sich diese gelben Schollen aufquellend in neblig trübe Kugeln krümligen Inhalts, die ähnlich aussehen wie in Zerfall begriffene Fettkörnchenzellen. Durch Aether, Chloroform und Schwefelkohlenstoff werden diese gelben Körper ziemlich rasch geIöst. Durch Carmin wird auch bei längerer Behandlung die grosse Mehrzahl dieser Fettschollen nicht tingirt. Einzelne jedoch färben sich hellcarminroth. Behandlung mit Jod und Schwefelsäure bewirkt keine, oder keine merkliche Veränderung. Sie zeigen gegen Fäulniss eine bedeutende Resistenz; vor einem halben Jahre wurde mir ein bereits in schmierig fanlendem Zerfall begriffenes Hundehirn zur Untersuchung zugesandt, in dem sich die genannten Fettkörper in ganz unverändertem Zustande vorfanden. An Schnittpräparaten, die (nach Härtung) in absolutem Alcohol entwässert und in Creosot oder Nelkenöl aufgestellt worden, sieht man die Fettschollen nicht mehr, wenigstens nicht mehr deutlich.

Diese Fettschollen nun zeigen sich in ganz ungeheurer Menge. Man trifft sie durch's ganze Gehirn und Rückenmark hindurch, die Gefässe begleitend und zwar vorwiegend die kleineren Gefässe und die Capillaren, oft gehäuft in grösseren Mengen beisammen, nicht selten die kleinen Gefässe wie Beeren einen Traubenkamm umgebend. An den Capillaren scheinen sie einfach aufzukleben, in den grössern Gefässen liegen sie deutlich intra adventitiam. Sie liegen also ebenfalls, wenigstens vorwiegend, in den perivasculären Räumen der Gefässe.

Ich habe bis jetzt, wenn ich Grösse und Form der betreffenden Fettkörper und ihre Häufigkeit bei Lyssa besonders in's Auge fasse, keine ähnlichen Bildungen bei irgend einem andern pathologischen Process der Nervencentren des Hundes und des Menschen, noch viel weniger an normalen Gehirnen gesehen. Ich betone in dieser Beziehung in's Besondere, dass bei nervöser Stanpe der Hunde, welche nach meinen Untersuchungen mit myelitischen Processen einhergeht, sich der betreffende Fettkörper nicht findet.

Ich fühle mich daher versucht, diesen Fettkörper, mit Rücksicht namentlich auf seine massenhafte Anhäufung, als pathognomonisch für den Lyssaprocess anzusprechen. Erweist sich diese Anschauung als richtig, so wäre damit auch dem weniger Geübten Gelegenheit geboten, in zweifelhaften Fällen von Lyssa aus dem pathologischen Befunde mit Hülfe des Mikroskops ein sicheres Urtheil über den Krankheitsprocess zu gewinnen, was bei der Unsicherheit der bis jetzt für die betreffende Krankheit bekannten Sectionsbefunde 
Ueber die Veränderungen d. Gehirns u. Rückenmarks bei Lyssa.

nicht ohne praktische Bedeutung für die forensische Thierarzneikunde wäre.

Es unterliegt wohl keinem Zweifel, dass dieser Fettkörper mit der „hyaloiden Substanz und den Pigmentschollen und Pigmenthüllen“ identisch ist, wie sie Benedikt und Kolesnikoff beschrieben, oder mit der „mattglänzenden, stark lichtbrechenden, hyaloiden Substanz“ Was silieff's.

Dass die genannten Forscher die fraglichen Körper nicht als Fettkörper erkannt haben, findet seinen Grund möglicher Weise darin, dass sie ihre Untersuchung vorwiegend an gehärteten Präparaten angestellt haben.

\section{B. \\ Untersuchung der gehärteten Präparate.}

Von den gehärteten Präparaten wurden zwei und zwar ein in Alcohol und ein in Lösung von doppeltchromsaurem Kali gehärtetes Hirn - Rückenmark ganz vollständig, d. h. in fortlaufender Reihe von Schnitten untersucht. Bei den übrigen Präparaten erstreckte sich die Untersuchung nur auf die bei dem vorliegenden Krankheitsprocess wesentlich afficirten Partien; d. h. es wurden je aus dem Lenden-, Brust- und Halsmark und aus dem verlängerten Mark eine grössere und aus den übrigen Partien des Gehirns nur eine geringe Anzahl von Schnitten gefertigt. Die Schnitte wurden nach der gewöhnlichen Methode behandelt, d. h. nach der Tinction in absolutem Alcohol entwässert, in Creosot aufgehellt und in Sandarak oder Canadabalsam eingeschlossen. Nur wenige Schnitte wurden in Glycerin conservirt. Als Tinctionsmittel verwandte ich Carmin und Hämatoxylin.

Die Untersuchung nun der gehärteten Objecte ergab zunächst wieder im Wesentlichen die Befunde, wie wir sie schon durch die Untersuchung frischer Präparate constatirt hatten, nämlich Hyperämie und Exsudation von weissen Blutkörperchen in die perivasculären Lymphräume. Dagegen erscheint, wie schon bemerkt, in den entwässerten und mit Creosot aufgehellten Schnitten jener eigenthümliche Fettkörper verschwunden. Derselbe findet sich jedoch auch nach der Härtung in Zerzupfungspräparaten und in einfach in Glycerin aufgehellten Schnitten. Es ist wohl die Annahme gerechtfertigt, dass der betreffende Fettkörper durch die Behandlungsmethode theilweise oder ganz gelöst, oder sonst unsichtbar gemacht wird. Diese Annahme wird durch den Umstand unterstützt, dass man an mit Carmin tingirten, in Creosot aufgehellten Schnitten in den Gefässwandungen, insbesondere deren adventitiellen Partien, häufig helle Vacuolen von 
rundlicher bis ovaler Gestalt findet, welche in ihrer Grösse und Form so ziemlich mit jenen Fettkörpern und Fettkörperhaufen übereinstimmen, die an frischen Gefässen so zahlreich entgegentreten. Ich glaube diese Vacuolen als die Residuen der beschriebenen Fettkörper ansprechen zu müssen.

Was die Hyperämie und die perivasculäre Exsudation anbetrifft, so constatirt die Untersuchung von Schnittpräparaten in Bezug auf Intensität und locale Ausbreitung der Affection im Allgemeinen, was wir bereits bei der Untersuchung frischer Präparate gefunden haben.

Die Hyperämie findet sich durch das ganze Rückenmark und das ganze Gehirn verbreitet, am ausgesprochensten im verlängerten Mark und im Rückenmark, am mässigsten in dem Kleinhirn und in den Hemisphären des Grosshirns.

Aehnlich verhält es sich mit der perivasculären Exsudation. Dieselbe tritt zwar auch in den Hemisphären des Grosshirns und im Kleingehirn an einzelnen Gefässen noch in ausgeprägtem Grade auf. Die Mehrzahl der Gefässe zeigt aber hier die betreffende Affection nur sehr spärlich oder gar nicht. Viel ausgedehnter findet sich die Exsudation schon im Corp. striat., im Thalam. opt., im Linsenkern, in den Vierhügeln und in der Ponsgegend. Fast ganz allgemein, die grosse Mehrzahl der Gefässe beschlagend, erscheint sie in der Medulla oblongata und lässt sich von hier nach unten, durch das ganze Rückenmark an Intensität nur wenig abnehmend, verfolgen.

Im Weitern aber fand ich in all den verbreiteten Präparaten nicht selten capilläre Apoplexien und zahlreiche anderweitige Veränderungen der Substanz, die ich als entzündliche Herde bezeichnen muss.

Was zunächst die capillären Apoplexien anbetrifft, so zeigten sich dieselben in den verschiedenen Präparaten in ungleicher Häufigkeit. Sie fanden sich aber in allen fast ausschliesslich nur im verlängerten Mark und im Rückenmark, also da, wo, wie wir gesehen haben, auch die Hyperämie am stärksten ist. Die apoplectischen Herde sind von sehr wechselnder Ausdehnung. Sie betreffen fast ausschliesslich nur die graue Substanz, das Gewebe infiltrirend oder anseinanderdrängend und erscheinen sämmtlich ziemlich frisch. In pigmentöser Umwandlung begriffene Apoplexien findet man nicht. In Fig. 5 sehen wir ein solches ganz colossales Extravasat im Hinterhorn des Halsmarkes, das Gewebe auseinanderdrängend und zum Theil infiltrirend; inmitten des Extravasates (bei A.) flottirt ein Theil des zerrissenen Gefässes. Die mit B. bezeichneten Partien stellen durchschnittene Gefässe dar. 
Der allfällige Einwand, dass stark erweiterte und strotzend gefüllte. Gefässe diese Apoplexie vortäuschen dürften, widerlegt sich nicht allein durch das Fehlen von Gefässwandungen und die oft bedeutende Ausdehnung der Blutmassen, sondern auch durch die Erscheinung, dass die rothen Blutkörperchen deutlich die Randzone des durch das Blut auseinandergedrängten Gewebes durchsetzen.

Die entzündlichen Herde stellen sich dar als 0,3-1,0 Mm. im Durchmesser haltende, mehr weniger dichte Anhäufungen von „Kernen“. Besonders schön und deutlich markiren sich diese Herde durch Hämatoxylintinction (Fig. 2 und 4). Weniger übersichtliche und klare Bilder liefert die Tinction mit Carmin (Fig. 3). Letztere Tinctionsmethode hat aber den Vortheil, dass sie uns über die Natur jener Kerne eine deutlichere Auschauung verschafft. Dieselben erscheinen an Schnitten, die mit Carmin tingirt sind, nach Grösse, Form und Aussehen identisch mit weissen Blutkörperchen - völlig analog jenen Elementen, aus welchen die perivasculäre Exsudation besteht. (Fig. 2C., Fig. 3, 4, 5.)

Häufig ist die Anhäufung dieser lymphoiden Elemente eine so dichte, dass Kern an Kern zu liegen scheint, stellenweise wieder ist die Infiltration eine mehr diffuse. Immer aber tritt sie auf Querschnittspräparaten in deutlichen Herden auf. Was wir bereits über die Localisation der perivasculären Exsudation gesagt, gilt im Allgemeinen auch für die Verbreitung der Entzündungsherde. Wir haben aber in Bezug auf diesen Punkt noch Folgendes beizufügen: Zunächst ist zu bemerken, dass wir Kleinhirn- und Grosshirnhemisphären von den beschriebenen entzündlichen Infiltrationen fast vollständig frei fanden. Im Kleinhirn habe ich solche gar nicht gesehen und in den Hemisphären des Grosshirns im Ganzen nur wenige.

Die zahlreichsten und ausgedehntesten Entzündungsherde fanden sich in allen meinen Präparaten in der Medulla oblongata (Fig. 2 und 3); von hier lässt sich der Entzündungsprocess nach oben, sc. nach vorn, an Intensität und Häufigkeit abnehmend, durch die Vierb ügel- und Haubengegend bis in die basalen Ganglien verfolgen. Im Rückenmark selbst ist die Infiltration bald in ziemlich gleichmässiger Intensität bis in's Lendenmark herab zu constatiren, bald betrifft sie in vorwiegender Intensität nur das Halsmark und das obere Brustmark oder auch das Brustmark ist relativ frei von der Entzündung, welche nur Halsmark und Lendenanschwellung beschlägt.

Im Weitern zeigt sich, dass in den beschriebenen, von dem Processe vorzüglich befallenen Partien, die Entzündung sich in ganz auffallender Weise vorwiegend in der grauen Substanz localisirt. 
Ferner lässt sich leicht constatiren, dass der Entzündungsprocess in seiner Verbreitung mit Vorliebe dem Verlaufe der Gefässe folgt. Die Herde befinden sich durchgehends um Gefässe herumgelagert und speciell um solche Gefässe, deren Lymphraum von der entzündlichen Exsudation ebenfalls stark betroffen ist, so dass man sich kaum der Auffassung entschlagen kann, dass die Entzündungsherde durch Auswanderung von weissen Blutkörperchen aus den Gefässen, über den perivasculären Raum hinaus, in das Gewebe entstanden sind und gewissermassen eine zeitliche und locale Fortsetzung der perivasculären Auswanderung, eine Consequenz des letzterer zu Grunde liegenden Krankheitsprocesses darstellen. Obiges Verhalten dürfte wohl auch die Prädilection erklären, mit welcher der Entzündungsprocess die grane Substanz befällt, die bekanntlich durchschnittlich viel gefässreicher ist, als die weisse.

Nach dem Gesagten muss der Process als eine vom Gefäss-Bindegewebsapparat ausgegangene Entzündung aufgefasst werden.

Er unterscheidet sich von der gewöhnlichen Myelitis, sc. Encephalitis, dadurch, dass die durch die Entzündung gesetzte Ernährungsstörung bei Weitem nicht den Grad erreicht, wie bei jenen Krankheitsprocessen. Bis zur Erweichung der betreffenden Gewebspartien kommt es bei der Lyssa nicht. Die Gewebe zeigen keine deutlich nachweisbare Destruction. Makroskopisch bieten Querschnitte gehärteter Präparate nur ganz geringe Anomalien. Man sieht allerdings mit unbewaffnetem Auge dentlich die Hyperämie. Man sieht auch an Querschnitten durch das verlängerte Mark die Entzündungsherde als dunklere Punkte markirt. Ausgedehntere Verfärbungen, wie solche bei eigentlicher Myelitis gewöhnlich, sind makroskopisch nicht sichtbar.

Der Gang meiner Untersuchung brachte es leider mit sich, dass ich von frischen Präparaten nur die Gefässe einer speciellen Untersuchung unterwarf. Ich habe später, nachholend, aus einer in chromsaurem Kali conservirten Medulla oblongata Zerzupfungspräparate in Liq. Kali caust. angefertigt und dabei nicht sehr zahlreiche, aber deutliche Fettkörnchenzellen zur Anschauung gebracht.

Im Uebrigen ergab meine Untersuchung nur wenig nennenswerthe Veränderungen der Neuroglia und ảer nervösen Elemente selbst. Die Ganglienzellen des Rückenmarks, speciell der Vorderhörncr ermangeln in den meisten meiner Präparate deutlicher Fortsätze und ihr Protoplasma zeigt häufig undeutlich verschwommene Contouren. Die Kerne derselben erscheinen durchgehends von normalem Aussehen. Mitunter 
Ueber die Veränderungen d. Gehirns u. Räclrenmarks bei Lyssa. 503

sieht man ein bis drei lymphoide Kerne in dem Protoplasma der Ganglienzellen. An den Nervenfasern selbst konnte ich mit Sicherheit keine Veränderungen nachweisen. Mit Carmin tingirte Schnitte aus den basalen Ganglien und dem Stabkranz eines der von mir untersuchten Lyssagehirne zeigten ziemlich zahlreiche Spinnenzellen. An entsprechenden Schnitten der anderen Präparate sah ich diese Gebilde nicht.

Wenn nun auch unsere Untersuchung kaum nennenswerthe secundäre Veränderungen der Nervenelemente der. Centralorgane zur Darstellung gebracht hat, so dürfte doch ein Umstand darauf hinweisen, dass mit dem beschriebenen Entzündungsprocess eine erhebliche Ernährungsstörung und Degeneration der Nervenelemente einhergeht. Es ist dies die so massenhafte Anhäufung jenes oben beschriebenen Fettkörpers in den perivasculären Räumen. Derselbe kann wohl kaum anders, denn als Resorptionsproduct, aufgefasst werden.

Ich muss hier zur Widerlegung allfälliger Einwände, die gegen meine Deutung der gewonnenen Bilder gemacht werden könnten, bemerken, dass ich zur Controle zahlreiche Schnitte aus normalen Hundehirnen nach genau derselben Methode behandelt habe und dass dieselben weder die perivasculäre Auswanderung, noch eine Infiltration des Gewebes mit Kernen zeigen, wie wir solche bei der Lyssa sehen. Ebenso ist es ganz unmöglich, die „Entzündungsherde“ als intra adventitiam liegend zu deuten, sobald man eine fortlaufende Reihe von Querschnitten sorgfältiger Betrachtung unterzieht. Man findet dann, dass in denjenigen Bezirken der Nervencentren, welche von der Entzündung besonders betroffen sind, z. B. in der grauen Commissur des Rückenmarks, die Infiltration sich oft auf weite Strecken, durch viele Gefässbezirke hindurch ziemlich gleichmässig fortsetzt; dieses Verhalten lässt sich auch sehr schön an Längsschnitten durch die betreffenden Prädilectionsstellen zur Anschauung bringen.

Ich erlaube mir dem Gesagten noch beizufügen, dass mit Hämatoxylin tingirte Querschnitte durch das Rückenmark von Hunden, welche an nervöser Staupe zu Grunde gegangen sind, ganz ähnliche Bilder lymphoider Infiltration zeigen, wie wir solche in Fig. 3, 4 und 5 dargestellt haben, nur sind bei Staupe die Herde z. Th. noch viel ausgedehnter. Bei der Staupe aber charakterisirt sich der Process als eine acute Myelitis in optima forma.

Es bleibt nun noch übrig, uns etwas specieller über die Localisation der Entzündungsherde zu verbreiten. 


\section{Rückenmark.}

Im Rückenmark finden wir fast ganz ausschliesslich die graue Substanz von der Entzündung betroffen. Nur ganz ausnahmsweise trifft man auch kleine Herde mitten in der weissen Substanz. Etwas häufiger sieht man einen die Randpartien der Vorder- oder Hinterhörner einnehmenden Entzündungsherd sich noch etwas in die angrenzenden Markstränge fortsetzen. Aber auch diese Bilder sind im Ganzen selten.

In der grauen Substanz selbst ist die Entzündung eine sehr ausgesprochene. Ueber ihre Ausbreitung im Allgemeinen haben wir schon oben gesprochen. Was ihre Localisation, auf den Querschnitt projicirt, betrifft, so macht sich ein ziemlich gesetzmässiger Typus in der Verbreitung geltend. Es sind nämlich vorwiegend afficirt:

1. Die Partien um den Centralkanal, sc. die Gegend der grauen Commissur, (Fig. 4.)

2. Die Vorderhörner, insbesondere deren Randzone.

3. Die äussere Randzone der Hinterhörner.

Um den Centralkanal findet man fast auf allen Querschnitten eine mehr weniger ausgedehnte lymphoide Infiltration, welche die colossal erweiterten und strotzend mit Blut gefüllten Gefüsse (Venen) umlagert und bald nur die Umgebung der Gefässe beschlägt, bald den grössten Theil der Commissuren einnimmt. (Fig. 4 stellt einen solchen Querschnitt durch die Gegend des Centralkanals dar. - Das Präparat ist der Halsanschwellung entnommen.)

Es setzt sich die Entzündung um den Centralkanal ziemlich ununterbrochen durch das ganze Halsmark, resp. das ganze Rückenmark, fort. Die Bezeichnung herdartiger Infiltration hat eigentlich nur ihre rolle Berechtigung in Beziehnng anf den Querschnitt. Vom Längsschnitt aus betrachtet, erscheint die Affection als eine mehr weniger diffuse. Fast ebenso constant sind die Vorderhörner betroffen, vorwiegend deren Randzone. Bald ist die innere Partie, bald die äussere Randzone stärker infiltrirt. Im Ganzen lässt sich auch hier wieder constatiren, dass die Entzündung dem Verlauf der mit den vordern Wurzelfasern eintretenden grösseren Gefässen folgt.

Ebenso verhält es sich mit den Hinterhörnern, wo übrigens die Entzündung viel weniger constant ist. Aueh hier begleitet sie die grössern Gefässzüge. Abgesehen von den genannten constant oder sehr häufig afficirten Prädilectionsstellen der Entzündung, finden sich aber kleinere und grössere Herde auch da und dort in den übrigen 
Partien der grauen Substanz, meist deutlich nachweisbar um grössere Gefässe herumlagert.

Nicht selten stösst man in Rückenmark auf capilläre Apoplexien. Dieselben lassen in Bezug auf ihre Localisation keine Gesetzmässigkeit constatiren. Nur finden auch sie sich fast ausschliesslich in der grauen Substanz. (Fig. 5.)

Eine besondere Beachtung verdient der Centralkanal und dessen Verhalten zu der Entzündung. Durchgehends ist derselbe stark erweitert und mit einem eigenthümlichen Exsudat gefüllt, das von mehr weniger zahlreichen, lymphoiden Elementen durchsetzt ist. Dieses Exsudat stellt sich in den aufgehellten Schnitten als eine gleichmässig opake Masse dar, die durch Carmin und Hämatoxylin blass tingirt wird. Das Exsudat findet sich aber auch sehr hänfig ausser dem Centralkanal, in dessen Umgebungen, insbesondere um die daselbst nie fehlenden, grösseren Venenstämme herum.

\section{Medulla oblongata.}

(Fig. 2 und 3.)

Wie schon bemerkt ist die Medulla oblongata von dem Entzündungsprocess am stärksten betroffen. Auf Querschnitten derselben fallen zunächst wieder die erweiterten strotzend gefüllten und hier in der grossen Mehrzahl durch perivasculäre Exsudation stark veränderten Gefässe auf. Im Weitern zeigen Querschnitte zahlreiche kleinere und grössere Entzündungsherde. Dieselben folgen auch hier dem schon wiederholt besprochenen Typus. Sie localisiren sich, wenn auch nicht ausschliesslich, doch vorzugsweise in der grauen Substanz und zwar insbesondere in der Nachbarschaft des vierten Ventrikels und in der Raphe (Fig. 3). Die ausgedehntesten und häufigsten Herde trifft man in den Kernen des Glossopharyngens, Vagus und Accessorius. Verfolgt man Schnitt an Schnitt, so zeigt sich, dass die Entzündung sich diffus fast durch die ganze Ausdehnung dieser Kerne hindurchsetzt, allerdings in sehr variirender Intensität, d. h. mit wechselnder Dichtigkeit der das Gewebe infiltrirenden Entzündungskerne.

In der Raphe begleitet die lymphoide Exsudation vorwiegend die hier verlanfenden grossen Gefässe. Die weisse Fasermasse ist im Ganzen nur sehr wenig von der Entzündung afficirt und ebenso zeigen die Oliven nur unbedeutende Veränderungen. 


\section{Ponsgegend.}

In der eigentlichen Ponsfaserung habe ich nur ganz selten kleine Herde gesehen. Ebenso sind die Fasern der Pedunculi, sc. Pyramiden, in der Ponsgegend fast vollständig von dem Entzündungsprocess verschont. Dagegen finden sich zalreiche und mitunter ausgedehnte Herde in den Kernen des Acusticus, des Facialis und des Abducens.

Wenig afficirt erscheint auch hier die Faserung der Pedunculi und die Bindearmgegend.

\section{Vierhügel.}

Auch hier finden sieht man ziemlich zahlreiche Herde entzündlicher Infiltration und zwar insbesondere in der grauen Substanz der untern und obern Vierhügel selbst und in der Nachbarschaft des Aquae duct. Sylvii.

\section{v. Thaiamus opticus. Linsenkern und Corpus striatum.}

In den grossen basalen Hirnganglien sehen wir, wenn wir die Reihe der Schnittpräparate von hinten nach vorn verfolgen, die entzündlichen Erscheinungen gradatim abnehmen. Die encephalitischen Herde werden seltener und verlieren an Ausdehnung, um schliesslich fast vollständig zu verschwinden. Doch lassen sie sich bis gegen das Ende des Thalam. optic. verfolgen. Einen gesetzmässigen Typus der Localisation in dieser Gegend habe ich bei Vergleichung der verschiedenen Präparate nicht nachweisen können.

\section{Hemisphären des Grosshirns.}

In den Hemisphären des Grosshirns begegnet man mit Ausnahme der Riechlappen im Ganzen nur sehr wenigen der beschriebenen Entzündungsherde. In den Riechlappen dagegen habe ich in den meisten Präparaten wieder ziemlich zahlreiche und $z$. Th. ausgedehnte herdartige Infiltrationen des Gewebes mit lymphoiden Elementen gesehen.

Im Anschlusse an die vorliegenden eigenen Untersuchungen erlanbe ich mir noch einige Mittheilungen über ein von Herrn Prof. Huguenin in Zürich in letzter Zeit untersuchtes Gehirn eines an Lyssa verstorbenen Menschen. Herr Prof. Huguenin hatte die Güte mir seine Präparate und bezüglichen Notizen zur Benutzung zu überlassen.

Aus denselben ergiebt sich zunächst, dass auch bei Lyssa humana im Allgemeinen dieselben pathologischen Veränderungen im Gehirn und Rückenmark gesetzt werden, wie bei der Lyssa der Hunde. 
Ueber die Veränderungen d. Gehirns u. 'Rückenmarks bei Lyssa. 507

Auch in dem vom Prof. Huguenin untersuchten Präparate finden sich durch das ganze Rückenmark und die Medulla oblongata hindurch neben starker Hyperämie Exsudation von lymphoiden Elementen in die perivasculären Räume, capilläre Blutungen und diffuse entzündliche Infiltration des Gewebes; jener oben beschriebene eigenthümliche Fettkörper ist ebenfalls in grosser Menge vorhanden. Kurz wir haben im Allgemeinen dasselbe Bild pathologisch-anatomischer Veränderungen, wie wir es bereits beschrieben haben.

Dagegen zeigen sich in Bezug auf die Ausbreitung des Processes einige nicht unwesentliche Unterschiede.

Im Rückenmarke jenes an Lyssa verstorbenen Menschen fand sich die perivasculäre Exsudation und die entzündliche Infiltration von der Medulla oblongata an bis iu die Lendenanschwellung herab, fast ausschliesslich die graue Substanz beschlagend und an Intensität nach unten abnehmend. Die Entzündung tritt hier vorwiegend um den Centralkanal und in den Vorderhörnern auf, die grössern Gefässe begleitend. Im Centralkanal sieht man ziemlich dieselbe Veränderung, wie wir sie schon bei Lyssa canina kennen gelernt haben.

Die Medulla oblongata ist auch hier am, stärksten vom Entzündungsprocess ergriffen. Die bekannten entzündlichen Veränderungen sind am intensivsten in der Raphe und dann im Vagus-, Glossopharyngeus- und Accessoriuskern und zwar hier, wie dort, an Intensität nach oben abnehmend.

Die Gegend der Pyramidenkreuzung zeigt keine erheblichen Veränderungen. Soweit wäre die Localisation der Erscheinung ziemlich dieselbe, wie wir sie an von Hunden stammenden Gehirnen getroffen haben.

Dagegen erscheinen in dem von Huguenin untersuchten Gehirn Thalamus, Streifenhügel und Linsenkern frei von allen bezüglichen Veränderungen und ebenso sind die Pedunculi, die Querfaserung des Pons und die Vierhügel normal. Oculomotorius- und Trochleariskern sind ebenfalls nicht afficirt. Die ersten Veränderungen - Gefässerweiterungen mit Auswanderung - sehen wir, nach unten die Reihe der Querschnitte verfolgend, im motorischen Kern des Trigeminus. Fast ganz frei ist die Gegend des Facialiskerns und des Hypoglossuskerns.

In den Hemisphären des Grosshirns und Kleinhirns lassen sich in dem betreffenden menschlichen Gehirn keine wesentlichen Anomalien nachweisen.

Es ist also der Entzündungsprocess in dem vorliegenden menschlichen Gehirn in Bezug auf Ausbreitung ein viel beschränkterer, er- 
streckt sich nach oben kaum über die Medulla oblongata hinaus. Dagegen ist er gerade hier und im Halsmark im Ganzen sehr ausgesprochen und intensiver als bei den von mir untersuchten Präparaten an Lyssa verstorbener Hunde.

Was mir namentlich bei der Betrachtung der Schnittpräparate jenes Menschenhirns auffiel, war die grosse Ausdehnung der Entzündungsherde und die weit grössere Anzahl von capillären Apoplexien in der Medulla oblongata und im Halsmark.

Fassen wir die Ergebnisse unserer Untersuchungen in Kürze zusammen, so gelangen wir zu folgenden Resultaten und Schlüssen:

1. Die Wuthkrankheit localisirt sich in den Nervencentren in Form einer von dem Gefässapparat ausgehenden Entzündung. Diese Entzündung charakterisirt sich durch perivasculäre Exsudation und theils herdartige, theils mehr diffuse Infiltration des Gewebes mit lymphoiden Elementen. Zugleich tritt, wahrscheinlich als Produkt degenerativer Vorgänge in den Nervenelementen, massenhaft ein eigenthümlicher Fettkörper in den perivasculären Räumen auf.

2. Die Entzündung ist als das Anfangsstadium einer acuten Myelitis, sc. Encephalitis, aufzufassen.

Dass der Process nicht, wie bei der gewöhnlichen acuten Myelitis, bis zur Erweichung fortschreitet, findet seine Erklärnng in der kurzen Daner der Krankheit und die kurze Dauer selbst dürfte z. Th. abhängen von der Localisation des Entzündungsprocesses.

3. Dieser afficirt am stärksten die Medulla oblongata und hier speciell die Kerne des Glossopharyngeus, Vagus und Accessorius.

4. Beim Menschen scheint der Process sich auf Rückenmark und Medulla oblongata zu beschränken; bei der Wuthkrankheit der Hunde wird auch das Grosshirn von der Entzündung betroffen.

\section{Erklärung der Abbildungen.}

Fig. 1. Gefässramification aus der Vierhügelgegend. Lyssa, Hund. Vergrösserung: Hartnack Ocul. 3. Obj. 7.

A. Starke perivasculäre Auswanderung.

B. Zahlreiche Fettschollen,

Fig. 2. Lymphoide Infiltration, sc. Entzündungsherd aus dem Glossopharyngeuskern des Hundes. Hämatoxylintinction.

Hartnack Ocul. 3. Obj. 4.

A. und B. Gefässe. Bei A. starke perivasculäre Auswanderung.

C. Entzündungsherde. 
Ueber die Veränderungen d. Gehirns u. Rü̈ckenmarks bei Lyssa. 509

Fig. 3. Lymphoide Infiltration des Gewebes, aus den untern Partien der Medulla oblongata. Hund. Carmintinetion.

Hartnack Ocul. 3. Obj. 7.

Fig. 4. Querschnitt durch die Halsanschwellung des Rückenmarks. Hund. Hämatoxylintinction.

Hartnack Ocul. 2. Obj. 4.

A. Vordere Commissur.

B. Hintere Commissur.

C. Centralkanal durch Exsudation erweitert; in seiner Ungebung lymphoide Infiltration des Gewebes.

D. Querdurchschnittene Venen.

Fig. 5. Extravasation im Hinterhorn des Halsmarks. Hund. Carmintinction.

Hartnack Ocul. 3. Obj. 4.

A. Geborstenes Gefäss in dem Extravasat flottirend.

B. Gefässdurchschnitte. 
过
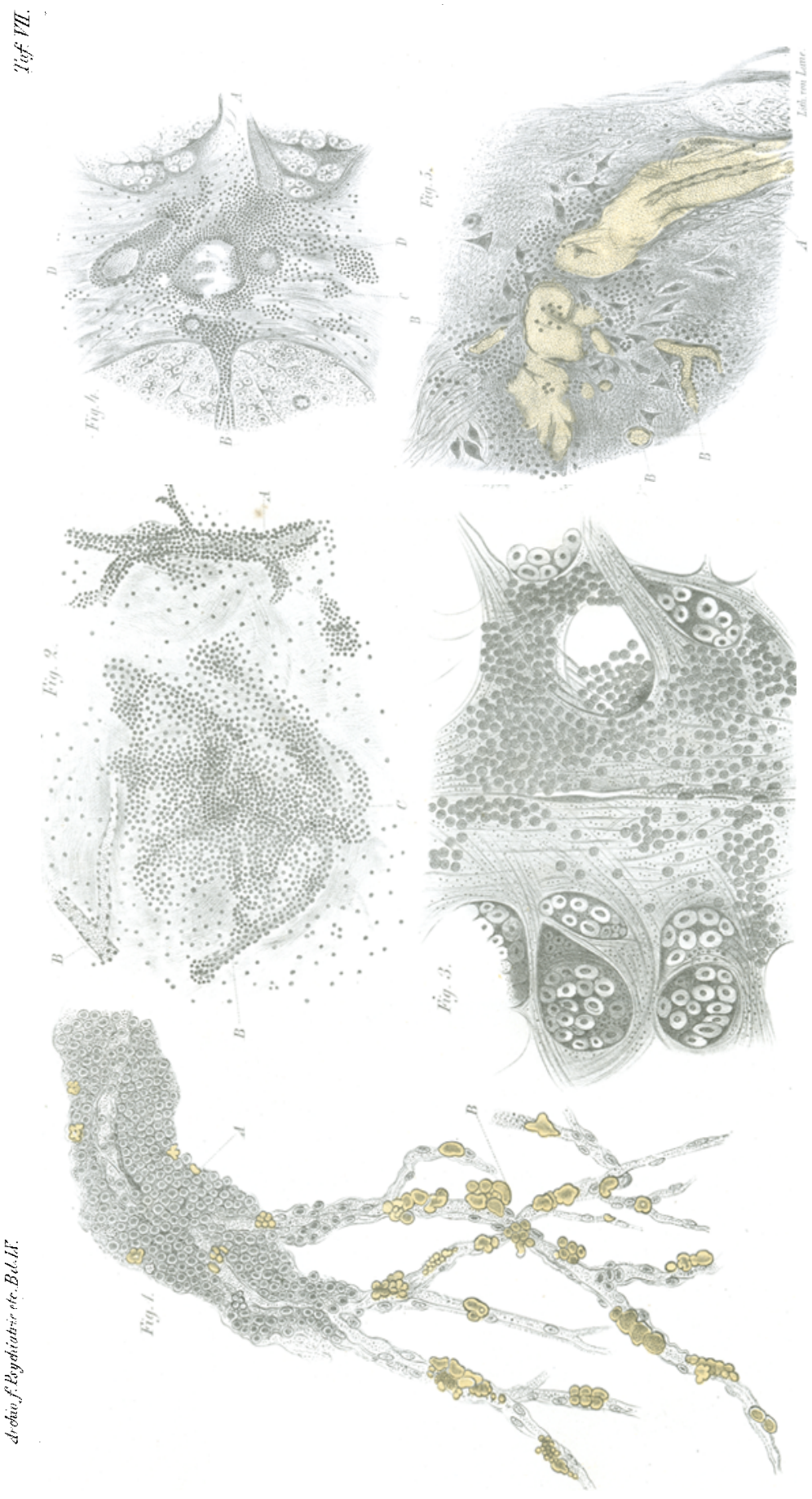